2010

\title{
An Analytical Model for Rotator Cuff Repairs
}

\author{
A. Aurora \\ Cleveland Clinic \\ Jorge E. Gatica \\ Cleveland State University, j.gatica@csuohio.edu
}

Antonie J. van den Bogert

Cleveland State University, a.vandenbogert@csuohio.edu

J. A. McCarron

Cleveland Clinic

Follow this and additional works at: https://engagedscholarship.csuohio.edu/enme_facpub

thleen A Derwin
Cleveland of the B. Bmechanical Engineering Commons, and the Biomedical Engineering and Bioengineering Commons

How does access to this work benefit you? Let us know!

\section{Publisher's Statement}

NOTICE: this is the author's version of a work that was accepted for publication in Clinical

Biomechanics. Changes resulting from the publishing process, such as peer review, editing,

corrections, structural formatting, and other quality control mechanisms may not be reflected in this document. Changes may have been made to this work since it was submitted for publication. A definitive version was subsequently published in Clinical Biomechanics, 25, 8, (01-01-2010); 10.1016/j.clinbiomech.2010.05.010

\section{Original Citation}

Aurora A, Gatica JE, van den Bogert AJ, McCarron JA, Derwin KA. (2010) An analytical model for rotator cuff repairs. Clinical Biomechanics 25(8): 751-758.

This Article is brought to you for free and open access by the Mechanical Engineering Department at EngagedScholarship@CSU. It has been accepted for inclusion in Mechanical Engineering Faculty Publications by an authorized administrator of EngagedScholarship@CSU. For more information, please contact library.es@csuohio.edu. 


\title{
An analytical model for rotator cuff repairs
}

\author{
A. Aurora ${ }^{\mathrm{a}, \mathrm{b}}$, J.E. Gatica ${ }^{\mathrm{b}}$, A.J. van den Bogert ${ }^{\mathrm{a}}$, J.A. McCarron ${ }^{\mathrm{c}}$, K.A. Derwin ${ }^{\mathrm{a}, \mathrm{c}, *}$ \\ a Department of Biomedical Engineering. ND2-20, Cleveland Clinic, 9500 Euclid Avenue, Cleveland, OH 44122, USA \\ ${ }^{b}$ Department of Chemical and Biomedical Engineering, SH 459. Cleveland State University, 2121 Euclid Avenue, Cleveland, OH 44115, USA \\ ' Department of Orthopedic Surgery. A41, Cleveland Clinic, 9500 Euclid Avenue, Cleveland, OH 44122, USA
}

\section{Introduction}

Rotator cuff tears affect $40 \%$ or more of those over age 60 (Milgrom et al., 1995; Tempelhof, 1999; Yamaguchi, 2001) and are a common cause of debilitating pain, reduced shoulder function and weakness. Thirty to seventy-five thousand rotator cuff repairs are performed annually in the United States (Vitale et al., 2007). While the best treatment for this disorder remains a topic of debate, open and arthroscopic surgical repair is currently the accepted "gold" standard for the treatment of tears that fail to improve after conservative treatment. Despite advances in the surgical treatment of these tears, high surgical failure rates that range from 20 to $90 \%$ have been reported (Accousti and Flatow, 2007; Bishop et al., 2006; Boileau et al., 2005; Galatz et al., 2004; Gazielly et al., 1994; Gerber et al., 2000; Harryman et al., 1991) due to factors not restricted to patient age, tear size and chronicity, muscle atrophy and degeneration, tendon quality, repair technique and the post-operative rehabilitation ( Bartolozzi et al., 1994; Cofield et al., 2001; Goutallier et al., 2003; Hamada et al., 1997; Iannotti,

* Corresponding author. Department of Biomedical Engineering, ND2-20. Cleveland Clinic, 9500 Euclid Avenue, Cleveland, OH 44122, USA.

E-mail address: derwink@ccf.org (K.A. Derwin).
1994; Riley et al., 1994; Romeo et al., 1999; Thomopoulos et al., 2002; Uhthoff et al., 2003). Hence, repair strategies that can augment the repair by mechanically reinforcing it, while at the same time biologically enhancing the intrinsic healing potential of the patient are needed.

Currently, natural and synthetic scaffolds are being used as devices to augment soft tissues repaired by sutures or suture anchors during the repair of large to massive rotator cuff tears (Aurora et al., 2007; Coons and Alan, 2006; Derwin et al., 2010). When used as an augmentation device, these scaffolds are believed to provide some degree of load sharing in a manner that will decrease the likelihood of tendon re-tear. While the biomechanical benefit achieved by using scaffolds as augmentation devices has recently been reported using human and animal models (Barber et al., 2008; Derwin et al., 2009; McCarron et al., 2010), no studies have investigated the degree of load sharing provided by a scaffold used for rotator cuff repair augmentation. Furthermore, the manner in which loads on an augmented rotator cuff repair are distributed among the various components of the repair is not known, nor is the relative biomechanical importance of the various components of the augmented rotator cuff repair. To answer these questions, the objectives of this study are to (1) develop quasi-static analytical models of simplified rotator cuff repairs, (2) validate the models by comparing the predicted model force to experimental measurements of force for human and canine rotator cuff 
repairs, and (3) use the models to predict the degree of load sharing provided by a scaffold used for rotator cuff repair augmentation. To achieve these objectives, analytical models were developed based on the physics of springs in series and parallel. The models were then validated and used to predict the degree of load sharing offered by a scaffold used for rotator cuff repair augmentation. Spring models allow us to predict the biomechanics (stiffness and yield load) of the repair construct during different loading scenarios and surgical repair techniques, which is not possible with finite element models that are used to predict the stress distribution in the tissues (Funakoshi et al, 2008; Sano et al, 2006, 2007; Seki et al, 2008; Wakabayashi et al., 2002). The development and validation of a spring model for rotator cuff repair is anticipated to provide an improved understanding of the mechanisms governing both nonaugmented and augmented rotator cuff repair biomechanics, which in turn might aid in the development of improved scaffolds and/or surgical techniques for rotator cuff repairs.

\section{Methods}

From the physical observation of non-augmented and augmented rotator cuff repairs, the individual components of the repair constructs were modeled as non-linear springs. Non-linear springs were used to model the points of compliance of the repair constructs, namely, the tendon attachment to bone, the tendon itself, the scaffold together with its attachment to bone and the scaffold attachment to the tendon. The parameters of the individual springs were estimated by non-linear least-squares analysis of the load-displacement data determined from isolated mechanical tests of each component of the repair. The assembly of the individual spring components into an aggregate model of the repair construct was based on the physics of springs in series and parallel. The estimated parameters were then used to solve the fully assembled model equations, and the force was predicted for the rotator cuff repair models. The models were validated by comparing the predicted model force to experimental measurements of force of human and canine rotator cuff repairs for a given displacement. The augmented rotator cuff repair model was then used to predict the degree of load sharing provided by the scaffold. Finally, a parametric sensitivity analysis was used to identify which of the component(s)/parameter(s) most influenced the mechanical behavior of the augmented rotator cuff repair model.

\subsection{Model structure}

\subsubsection{Non-augmented rotator cuff repair}

The non-augmented rotator cuff repairs in human (Fig. 1A) and canine (Fig. 1B) were modeled as two springs in series (Fig. 1C), namely, the bone-suture-tendon interface (spring\#1) and the tendon itself (spring\#2).

\subsubsection{Augmented rotator cuff repair}

The augmented rotator cuff repairs in human (Fig. 1D) and canine (Fig. 1E) were modeled as five springs in series and parallel (Fig. 1F). The tendon (spring\#2) was split into two half springs, spring\#2' and spring\#2". The bone-screw-scaffold-suture component (spring\#3) and the medial suture-tendon interface (spring\#4) were in series with each other and together in parallel with the primary tendon repair (springs 1 and $2^{\prime}$ ). The entire augmented rotator cuff repair model was then placed in series with the other half tendon spring\#2".

\subsection{Experimental mechanical testing}

All human and canine rotator cuff repairs described below were done using \#0 and \#2 Fiberwire sutures respectively (Arthrex Corporation, Naples, FL, USA).

\subsubsection{Non-augmented and augmented rotator cuff repairs}

2.2.1.1. Non-augmented rotator cuff repairs. Human $(n=5)$ and canine $(n=5)$ cadaveric shoulders were used to perform non-augmented rotator cuff repairs. For the human repairs, a strip of the superior infraspinatus tendon (12 mm wide) was released and repaired to the greater tuberosity using a double row transosseous technique with two Mason Allen sutures per row (Fig. 1A) (McCarron et al., 2010). Similarly, repair of the canine shoulders involved release and repair of the infraspinatus tendon to the greater tuberosity using two transosseous Mason Allen sutures (Fig. 1B).

2.2.1.2. Augmented rotator cuff repairs. The contralateral human $(n=5)$ and canine $(n=5)$ cadaveric shoulders were used to perform augmented rotator cuff repairs. For both human (Fig. 1D) and canine (Fig. 1E) specimens, a primary rotator cuff repair was performed as described above. The repairs were augmented with a $12 \mathrm{~mm} \times 35 \mathrm{~mm}$ prototypical polymer scaffold (X-Repair, Synthasome Inc., San Diego, CA, USA), fixed to the bone laterally with a screw and sutured medially to the tendon using three modified Mason Allen sutures (Derwin et al., 2009; McCarron et al., 2010).

For mechanical testing of all repairs, the muscle belly was freezeclamped and the repair was cycled between 5 and $100 \mathrm{~N}$ at $0.25 \mathrm{~Hz}$ and subsequently loaded to failure at $30 \mathrm{~mm} / \mathrm{min}$ (Derwin et al., 2009; McCarron et al., 2010). Optical markers placed in the bone and on the tendon were used to determine the displacement of the repair constructs. Experimental data for the non-augmented and augmented human rotator cuff repairs have been published previously (McCarron et al., 2010).

\subsubsection{Individual spring components}

2.2.2.1. Spring\#1 (bone-suture-tendon). The distance between an optical marker placed on the humeral head and in the tendon just medial to the repair sutures provided local displacements across the bone-suture-tendon interface. These displacement data were obtained from mechanical testing of human and canine non-augmented repairs ( $n=5$, respectively) (Fig. $1 \mathrm{~A}$ and $\mathrm{B}$ ).

2.2.2.2. Spring\#2 (tendon only). The distance between two optical markers placed on the tendon provided local displacements in the tendon. These displacement data were obtained from mechanical testing of human and canine non-augmented repairs ( $n=5$, respectively) (Fig. 1A and B). The displacements of spring\#2 were divided in half to obtain the displacements of spring\#2' and spring\#2" used in the augmented repair models.

2.2.2.3. Spring\#3 (bone-screw-scaffold-suture). A prototypic polymer scaffold was screwed to a wood block on one end and sutured with 3 simple stitches to a rod on the other end (Fig. 2A). The construct was preloaded to $5 \mathrm{~N}$ and subsequently loading to failure at $30 \mathrm{~mm} / \mathrm{min}$. The displacements of spring\#3 were obtained using actuator position from these isolated mechanical tests $(n=5)$.

2.2.2.4. Spring\#4 (medial suture-tendon). Three modified Mason Allen sutures were placed in either isolated human superior infraspinatus tendon or canine infraspinatus tendon and secured over a rod using a double half-hitch suture configuration (six throws) (Fig. 2B). The associated muscle was freeze-clamped and the suture interface was cyclically loaded $(5-30 \mathrm{~N}$ ) for 20 cycles at $0.25 \mathrm{~Hz}$ and subsequently loaded to failure at $30 \mathrm{~mm} / \mathrm{min}$. The displacements of spring\# 4 were obtained using actuator position from these isolated mechanical tests ( $n=5$, respectively). 

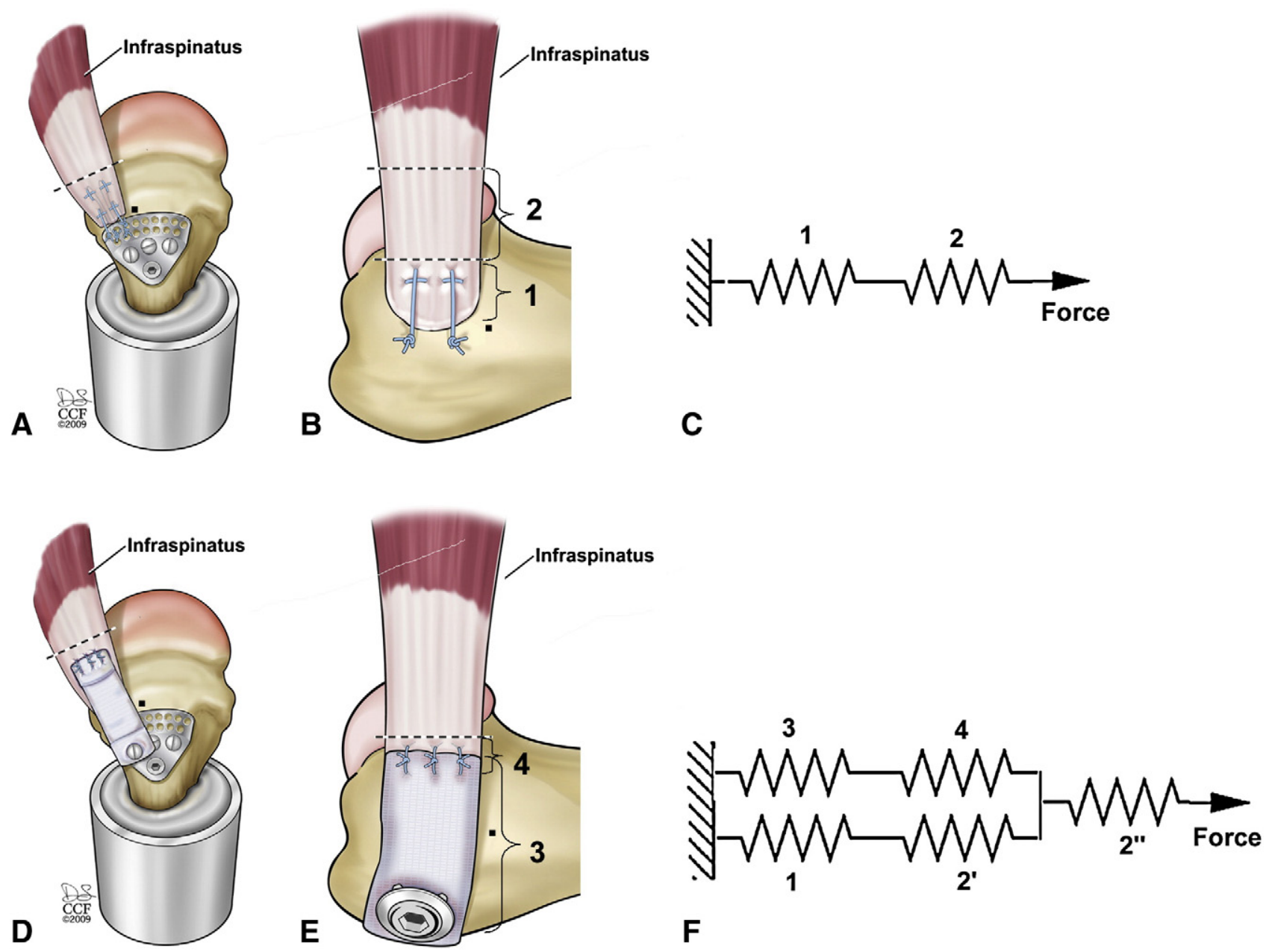

$\mathbf{F}$

Fig. 1. The non-augmented rotator cuff repairs in human (A) and canine (B) were modeled as two springs in series (C), namely, the bone-suture-tendon interface (spring\#1) and the tendon itself (spring\#2). The augmented rotator cuff repairs in human (D) and canine (E) were modeled as five springs in series and parallel (F). Details of the surgical repair techniques can be found in the text. Dotted lines represent suture markers that were placed on the tendon, and the black dot represents the optical marker that was placed on the bone, for optical displacement measurements.
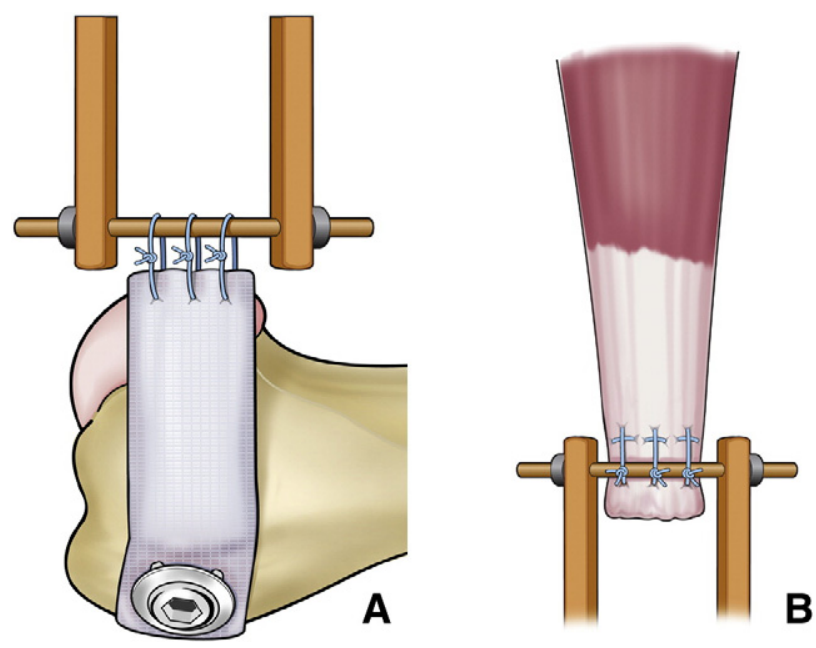

Fig. 2. Schematic of the experimental load-displacement testing for (A) spring\#3 (bonescrew-scaffold-suture) and (B) spring\#4 (medial suture-tendon interface). For spring\#3, the prototypic polymer scaffold was screwed to a wood block on one end and sutured with 3 simple stitches to a rod on the other end. For spring\#4, three modified Mason Allen sutures were placed in either isolated human superior infraspinatus tendon or canine infraspinatus tendon and secured over a rod using a double half-hitch suture configuration (six throws). Details of the mechanical testing protocol can be found in the text. The displacements of spring\#3 and spring\#4 were obtained using actuator positions.

\subsection{Formulation of the model}

The model was developed by representing the individual components of the repair as non-linear springs. Each non-linear spring was modeled using either a single-phase non-linear equation of the form $F=F^{o}+A x^{b}$ or a biphasic non-linear equation of the form $F=F^{o}+\frac{A x^{b}}{1+B x^{c}}$, depending on the equation goodness of fit. Here, $F^{o}, A, B, b$ and $c$ are parameters estimated by non-linear least-squares analysis of component specific experimental data. Single equations were then formulated for the non-augmented and augmented rotator cuff repair models using the physics of springs in series and parallel.

\subsection{Parameter estimation}

The parameters of the individual spring components were estimated by non-linear least square analysis of the component specific loaddisplacement data of the individual spring components up to the yield load to one of the aforementioned non-linear equations (Section 2.3). The yield load was defined as the first relative maximum load achieved during the experimental test. The non-linear least-squares analysis was performed using Sigma Stat.

\subsection{Model validation}

To predict the force response of non-augmented and augmented rotator cuff repairs, the fully assembled model equations were solved 
under static equilibrium conditions using standard non-linear equation solver, fsolve, provided with the optimization toolbox in MATLAB (Version 7.0). The model was validated by comparing the model predicted force to experimental measurements of the force of human and canine rotator cuff repairs for a given displacement. The 95\% confidence intervals for the model predictions were calculated using the error propagation formula

$E_{F, i}=\sum_{k=1}^{n}\left|\frac{\partial F}{\partial p_{k}}\right|_{x_{i}} E_{p, k}$

where, $E_{F, t}$ is the total error in the model equation at the displacement $x_{i}$ and $E_{p \cdot k}$ is the standard error of the $k$ th parameter. $\left.\frac{\partial F}{\partial p_{k}}\right|_{x_{i}}$ is the partial derivative of the model equation with respect to the $k$ th parameter evaluated at the displacement $x_{i}$.

The goodness of fit for each model was assessed using the root mean square error (RMSE) and the root mean square relative error (RMSRE) defined as follows:

RMSE $=\sqrt{\frac{\sum_{i=1}^{n}\left(F_{i}^{e}-F_{i}^{m^{2}}\right.}{n}} ;$ RMSRE $=\sqrt{\frac{\sum_{i=1}^{n} \varepsilon_{i}^{2}}{n}}$ where, $\varepsilon_{i}=\frac{F_{i}^{e}-F_{i}^{m}}{F_{i}^{e}}$

$F_{i}^{e}$ is the experimental measured force value, $F_{i}^{m}$ is the model predicted force for a displacement $x_{i}$ and $n$ is the number of data points. The predictions of the model were considered acceptable if the experimental data fell within the $95 \%$ confidence intervals of the predicted force response of the model and the RMSE (\% of the average experimental yield load) values of the model predicted force was less than or equal to $5 \%$. Five percent was chosen based on the consideration that, on average, model predictions could be reported with at least two significant figures.

\subsection{Parametric sensitivity analysis}

A parametric sensitivity analysis was used to identify which of the component(s)/parameter(s) most influenced the mechanical behavior of the augmented repair model. The sensitivity of each parameter was investigated by calculating the sensitivity coefficient $S$ defined as

$S_{p_{k}, x_{i}}=\left.\frac{\partial F}{\partial p_{k}}\right|_{x_{i}} \cdot\left|\frac{p_{k}^{o}}{F_{i}^{o}}\right| \forall i=1 \ldots N$

where $p_{k}^{o}$ and $F_{i}^{o}$ are the baseline values of the $k$ th parameter and the $i$ th force measurement respectively. The partial derivatives were estimated numerically by central differences. Our clinical interests and application of this model lie in predicting the change in the mechanics of rotator cuff repair with a change in surgical procedure and/or scaffold design. Hence, the parametric sensitivity analysis was carried out only for the parameters $A$ and $b$ of springs 1 , \#3 and \#4, which are considered to be associated with such modifications.

We now present the parameter estimation for the individual spring components, the model validation and predictions for non-augmented and augmented rotator cuff repairs, using both human and canine experimental data. The validated non-augmented and augmented repair models will then be compared to each other to investigate the mechanical role of scaffold augmentation. The distribution of forces in the individual components of the augmented repair will also be assessed through the model. This data will predict the degree of load sharing offered by using a prototype polymeric scaffold as an augmentation device for rotator cuff repairs. Finally, the results of the parametric sensitivity analysis will be presented.

\section{Results}

\subsection{Parameter estimation}

Table $1 \mathrm{~A}$ and $\mathrm{B}$ shows the parameters and the standard error of the individual spring components estimated for both human and canine rotator cuff repairs. Spring\#1 for both human and canine models and spring\#4 for the canine model were most reliably modeled using the biphasic non-linear equation (see Section 2.3). The other spring components in both human and canine rotator cuff repairs were most reliably modeled using the single-phase non-linear equation (see Section 2.3).

\subsection{Non-augmented repairs}

Fig. 3A and $\mathrm{C}$ show the experimental load-displacement data for the human and canine non-augmented repairs respectively, as well as the model predictions and the $95 \%$ confidence interval for the model predictions. The two spring non-augmented rotator cuff repair model appears to reliably reproduce the experimental data for both human and canine non-augmented rotator cuff repairs. Except for a small portion of the data corresponding to two human repairs at large displacement values, the experimental data remained within the $95 \% \mathrm{CI}$ limits, for both the human and canine models. The RMSE for the human nonaugmented rotator cuff repair model $(14 \mathrm{~N})$ is $8 \%$ of the average experimental yield load $(180 \mathrm{~N})$ and the RMSRE is $7 \%$. Ninety-seven percent of the model predictions for the human non-augmented rotator cuff repair can be reported with at least two significant figures. The RMSE for the canine non-augmented rotator cuff repair model ( $15 \mathrm{~N})$ is $11 \%$ of the average experimental yield load ( $140 \mathrm{~N})$ and the RMSRE is 12\% (Table 1C). Eighty-four percent of the model predictions for the

\section{Table 1}

Non-linear least-squares fit parameters (standard error) of the individual spring components for (A) human and (B) canine repairs. Significant figures were reported based on the magnitude of the standard errors, which represent $95 \%$ confidence level. "NS" implies that the parameter is not significantly different from zero at 95\% confidence level. Spring\#1 for both human and canine models and spring\#4 for the canine mode were most reliably modeled using the biphasic non-linear equation (see Section 2.3). The single-phase non-linear equation was the most reliable model for the other spring components in both human and canine repairs. (C) The root mean square error (RMSE) as a \% of the average experimental yield load, the root mean square relative error (RMSRE) and percent model predictions with at least two significant figures for human and canine rotator cuff repair models.

\begin{tabular}{|c|c|c|c|c|c|}
\hline Spring & $F^{o}$ & $A$ & $b$ & $B$ & c \\
\hline \multicolumn{6}{|c|}{ (A) Human model parameters } \\
\hline 1 & $20(10)$ & $300(200)$ & $2(1)$ & $2(1)$ & $2(1)$ \\
\hline $2^{\prime}$ & NS & $300(30)$ & $0.5(0.1)$ & $\mathrm{n} / \mathrm{a}$ & $\mathrm{n} / \mathrm{a}$ \\
\hline 3 & $-35(2.8)$ & $95(2.6)$ & $0.70(0.01)$ & $\mathrm{n} / \mathrm{a}$ & $\mathrm{n} / \mathrm{a}$ \\
\hline 4 & $-40(10)$ & $130(13)$ & $0.5(0.03)$ & $\mathrm{n} / \mathrm{a}$ & $\mathrm{n} / \mathrm{a}$ \\
\hline $2^{\prime \prime}$ & NS & $300(30)$ & $0.5(0.1)$ & $\mathrm{n} / \mathrm{a}$ & $\mathrm{n} / \mathrm{a}$ \\
\hline \multicolumn{6}{|c|}{ (B) Canine model parameters } \\
\hline 1 & $10(6)$ & $800(500)$ & $2(0.4)$ & $4(3)$ & $1.5(0.36)$ \\
\hline $2^{\prime}$ & NS & $350(46)$ & $1(0.2)$ & $\mathrm{n} / \mathrm{a}$ & $\mathrm{n} / \mathrm{a}$ \\
\hline 3 & $-35(2.8)$ & $95(2.6)$ & $0.70(0.01)$ & $\mathrm{n} / \mathrm{a}$ & $\mathrm{n} / \mathrm{a}$ \\
\hline 4 & $4(2)$ & $170(11)$ & $1.3(0.10)$ & $1.3(0.14)$ & $1.5(0.10)$ \\
\hline $2^{\prime \prime}$ & NS & $350(46)$ & $1(0.2)$ & $\mathrm{n} / \mathrm{a}$ & $\mathrm{n} / \mathrm{a}$ \\
\hline \multicolumn{6}{|c|}{ (C) RMSE and RMSRE for human and canine rotator cuff repair models } \\
\hline & & \multicolumn{2}{|c|}{ Non-augmented repair } & \multicolumn{2}{|c|}{ Augmented repair } \\
\hline & & Human & Canine & Human & Canine \\
\hline \multicolumn{2}{|c|}{ RMSE (N) } & 14 & 15 & 8 & 12 \\
\hline \multicolumn{2}{|c|}{ RMSE (\%) } & $8 \%$ & $11 \%$ & $3 \%$ & $6 \%$ \\
\hline \multicolumn{2}{|c|}{ RMSRE (\%) } & $7 \%$ & $12 \%$ & $19 \%$ & $14 \%$ \\
\hline \multicolumn{2}{|c|}{$\begin{array}{l}\text { Percent model } \\
\text { predictions with at } \\
\text { least two } \\
\text { significant figures }\end{array}$} & $97 \%$ & $84 \%$ & $93 \%$ & $85 \%$ \\
\hline
\end{tabular}


A

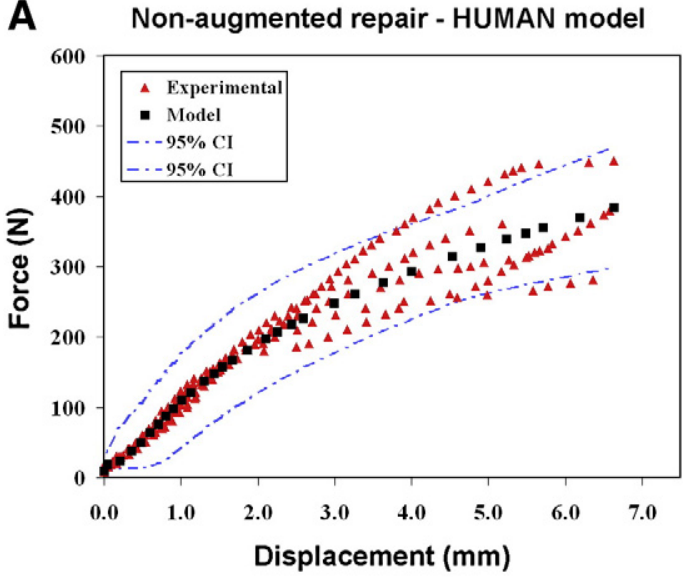

C

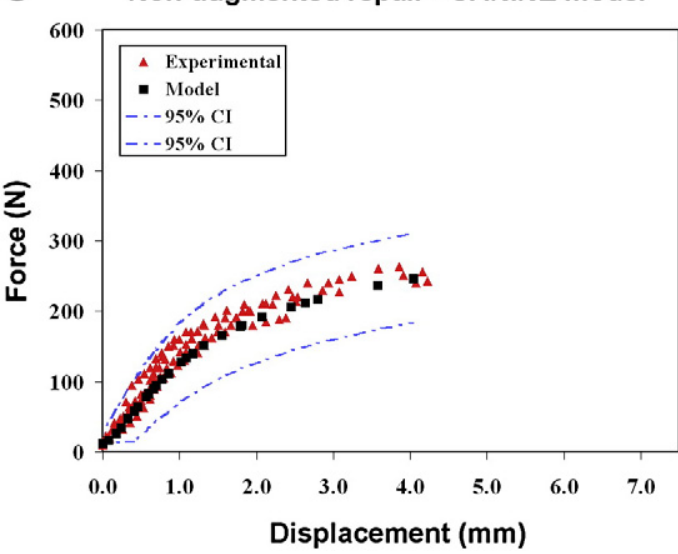

B

Augmented repair - HUMAN model

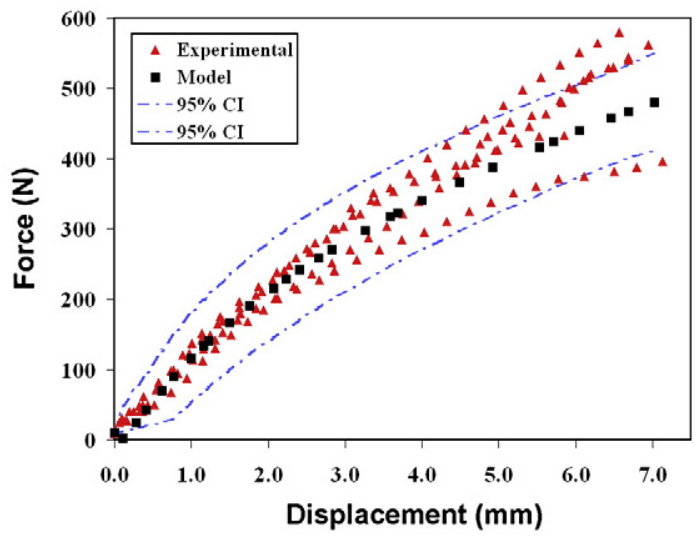

Augmented repair - CANINE model

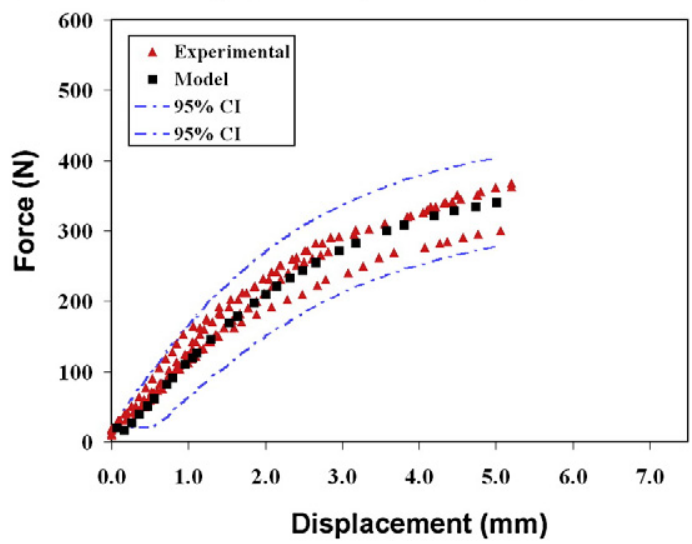

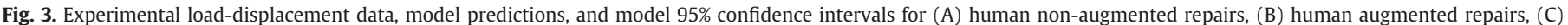

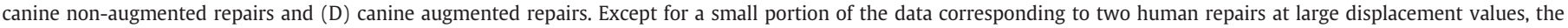
experimental data remained within the $95 \% \mathrm{CI}$ limits, for both the human and canine models.

canine non-augmented rotator cuff repair can be reported with at least two significant figures.

\subsection{Augmented repairs}

Fig. 3B and D show the experimental load-displacement data for the human and canine augmented repairs respectively, as well as the model predictions and the 95\% confidence interval for the model predictions. The five spring augmented rotator cuff repair model appears to reliably reproduce the experimental data for both human and canine augmented rotator cuff repairs. Except for a small portion of the data corresponding to two human repairs at large displacement values, the experimental data remained within the 95\% CI limits, for both the human and canine models. The RMSE for the human augmented rotator cuff repair model $(8 \mathrm{~N})$ is $3 \%$ of the average experimental load $(250 \mathrm{~N})$ and the RMSRE is $19 \%$. Ninety-three percent of the model predictions for the human augmented rotator cuff repair can be reported with at least two significant figures. The RMSE for the canine augmented rotator cuff repair model $(12 \mathrm{~N})$ is $7 \%$ of the average experimental yield load $(180 \mathrm{~N})$ and the RMSRE is $14 \%$ (Table 1C). Eighty-five percent of the model predictions for the canine augmented rotator cuff repair can be reported with at least two significant figures.

\subsection{Comparison of non-augmented versus augmented repair model}

Fig. 4A compares the model predictions for the non-augmented versus augmented rotator cuff repairs for the human model. The model suggests that scaffold augmentation will stiffen the repair construct, but only after displacements exceed $2 \mathrm{~mm}$. Further the model suggests that scaffold augmentation increases the yield load of the repairs. Similar results were found when comparing model predictions for the canine models (data not shown).

\subsection{Load sharing}

Fig. 4B shows the distribution of load between the augmentation components (spring\#3 and spring\#4) and the underlying tendon repair component (spring\#1 and spring\#2') as predicted by the model for human augmented rotator cuff repairs. The model suggests for displacements of $2 \mathrm{~mm}$ or less, the tendon repair component carries $\sim 80 \%$ of the total load acting on the augmented repair construct. Thereafter, the load carried by the tendon repair component is predicted to decrease from $80 \%$ to $73 \%$ of the total load. In other words, the augmentation component is predicted to carry between 20 and $27 \%$ of the total load acting on the augmented rotator cuff repair for the entire range of displacements. Similar results were found when comparing model predictions for canine augmented rotator cuff repairs (data not shown).

\subsection{Parametric sensitivity analysis}

The sensitivity analysis was carried out only for parameters $A$ and $b$ of springs\#1, \#3 and \#4, which may be considered to represent modifications associated with changes in surgical procedure and/or scaffold design. 


\section{A Non-augmented vs Augmented repair - HUMAN model}

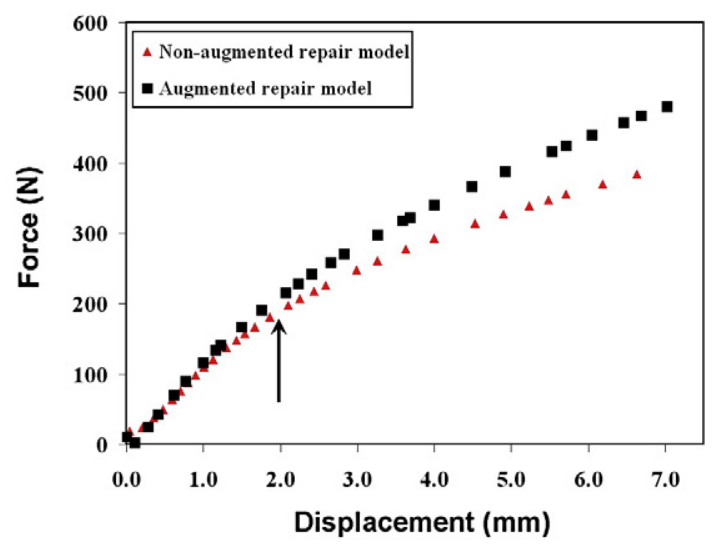

B Augmented repair - Load distribution

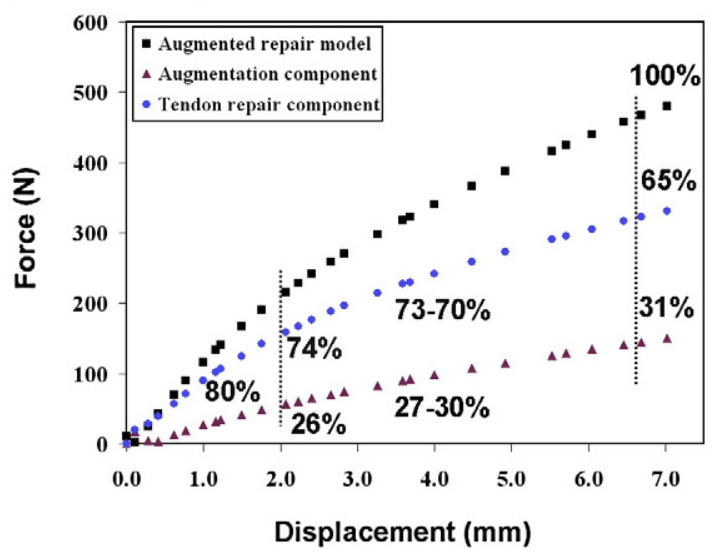

Fig. 4. (A) Model predictions for non-augmented versus augmented human rotator cuff repair. The model suggests that scaffold augmentation will stiffen the repair construct only after displacements exceed $2 \mathrm{~mm}$. Further, the model suggests that scaffold augmentation increases the yield load of the repair. (B) Load distribution in the different components of the augmented human rotator cuff tendon repair. The model predicts that the augmentation component will carry between 20 and $30 \%$ of the total load acting on the repair construct for the entire range of displacement.

\subsubsection{Parameter $A$}

Fig. 5A and $B$ show the parametric sensitivity coefficients for the parameter A corresponding to springs\#1, \#3 and \#4 for both human and canine augmented rotator cuff repair models. Both models are most sensitive to perturbations in parameter $A$ for spring\#1. A value of 0.5 for a given sensitivity coefficient suggests that a $100 \%$ change for the corresponding parameter from its baseline value $\left(p_{k}^{o}\right)$ would result in a $50 \%$ change in the model response from its baseline value $\left(F_{k}^{o}\right)$ (see Section 2.6). The model appears to be less sensitive to parameter $A$ in springs\#3 and \#4.

\subsubsection{Parameter $b$}

Fig. 5C and D shows the parametric sensitivity coefficients for the parameter $b$ corresponding to springs\#1, \#3 and \#4 for both human and canine augmented rotator cuff repair models. Similarly to the results observed for parameter $A$, both models are more sensitive to perturbations in parameter $b$ for spring\# 1 than for springs\#3 and \#4.

\section{Discussion}

There has been much interest in developing scaffolds as devices to augment the repair of large to massive rotator cuff tears. However, to date no studies have investigated the degree of load sharing provided by a scaffold used for rotator cuff repair augmentation. Furthermore, the manner in which loads on an augmented rotator cuff repair are distributed among the various components of the repair is not yet known. Finally, the relative biomechanical importance of various components of the rotator cuff repair construct is also unknown. Aiming to answer these questions, this study was designed to meet three objectives. The first objective was to develop quasi-static analytical models of simplified rotator cuff repairs, which was accomplished through formulating non-linear models based on the physics of springs in series and parallel. The second objective was to validate the models by comparing the model predicted forces to experimental measurements for human and canine rotator cuff repairs. Our results indicate that except for a small portion of the data corresponding to two human repairs at large displacement values, the experimental data remained within the $95 \% \mathrm{CI}$ limits, for both the human and canine models. The RMSE (\% of the average experimental yield load) of both human and canine augmented rotator cuff repair models was less than or equal to $6 \%$. The RMSE of both human and canine non-augmented rotator cuff repairs was slighter higher (8-11\%) as was the RMSRE of both human and canine rotator cuff repair models (7-19\%). The generally higher values for RMSRE arise primarily from large relative differences between the model predictions and experimental data at the low displacement measurements. However, more than $90 \%$ and $80 \%$ of the model predictions for the human and canine rotator cuff repair models respectively can be reported with at least two significant figures. This suggests that the models can provide a reliable prediction of the expected performance of the rotator cuff repairs. The models also predicted an increase in the yield load but not initial stiffness of repairs augmented with a prototypic polymeric scaffold, which is in agreement with the findings of our experimental repairs with this same scaffold (Derwin et al., 2009; McCarron et al., 2010). In summary, these results demonstrate the validity of the formulated models for predicting the biomechanics of these simplified human and canine rotator cuff repairs.

The final objective of the study was to predict the degree of load sharing provided by a scaffold used for rotator cuff repair augmentation. The model predicts that the augmentation component (i.e., the scaffold plus its attachments to tendon and bone) will carry between 20 and $30 \%$ of the total load acting on the repair construct for the entire range of displacement. A corollary to this result is that the underlying tendon repair carries the majority of the total load (70$80 \%$ ) acting on the augmented rotator cuff repair component for the entire range of displacement. This finding suggests that this particular scaffold, together with its attachments components, is less stiff than the tendon and its repair.

Our model appears to be most sensitive to perturbations in the parameters $A$ and $b$ of spring\#1 (the bone-suture-tendon interface). These results highlight the biomechanical importance of the suture attachment site, and suggest that the greatest improvements in the force carrying capacity of a tendon repair may be achieved by improving the bone-suture-tendon interface. At this time we are unable to explain the reversal in trend seen in the sensitivity curve of parameter $b$ for spring\# 1 for both human and canine augmented rotator cuff repair models, but this result may be related more to an interdependence among the model parameters than to the actual mechanics of the repair. We would like to emphasize that these model parameters do not carry any particular physical significance; rather they are derived from nonlinear least-squares analysis. However these parameters, particularly $A$ and $b$ for the individual components, are dictated by the shape of the load-displacement data and can be varied parametrically to simulate changes in the mechanical properties of each component. Such a parametric analysis will be the subject of our future work with this model.

Parameter $A$ for spring\#1, for both the human and canine models [Table $1 \mathrm{~A}$ ], has a large standard error compared to those of the remaining model parameters. We attribute this result to the inherent variability associated with performing a surgical repair. Further, spring\#1 for both human and canine models and spring\#4 for the canine model were most reliably modeled using the biphasic non-linear equation whereas all other 
A Parametric Sensitivity - 'A' (HUMAN model)

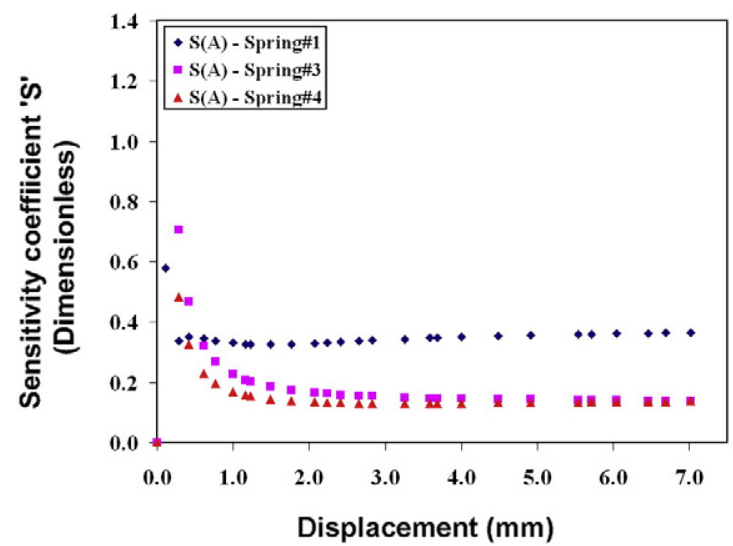

C Parametric sensitivity - 'b' (HUMAN model)

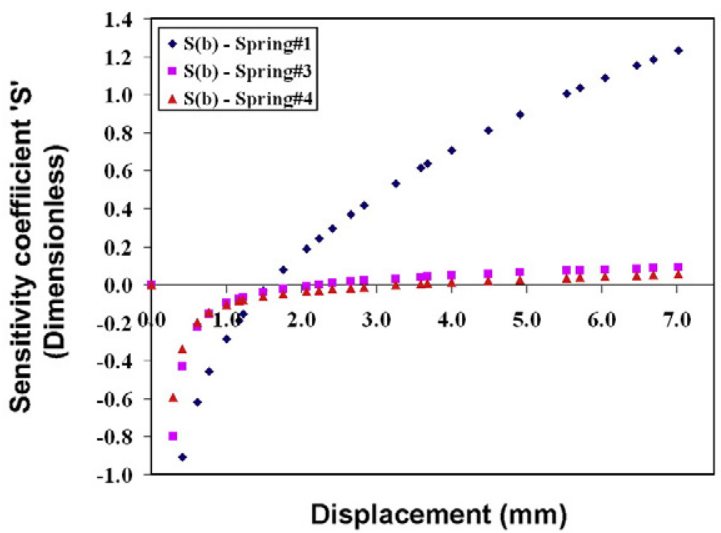

B

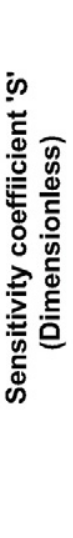

D

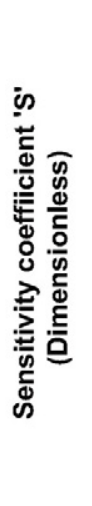

Parametric sensitivity - 'A' (CANINE model)

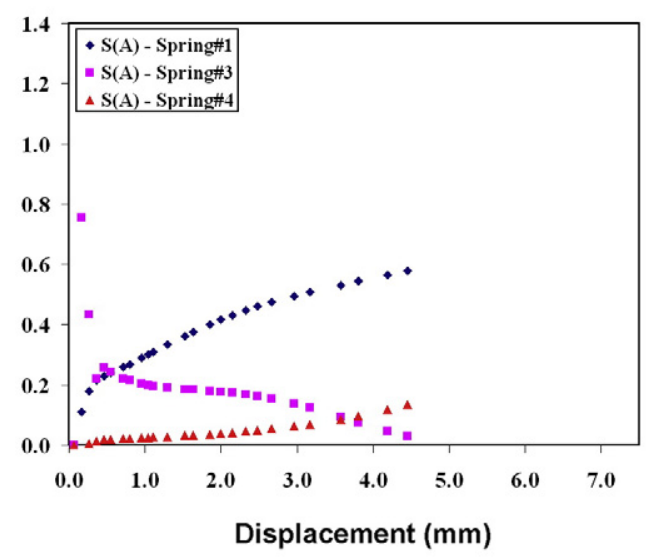

Parametric sensitivity - 'b' (CANINE model)

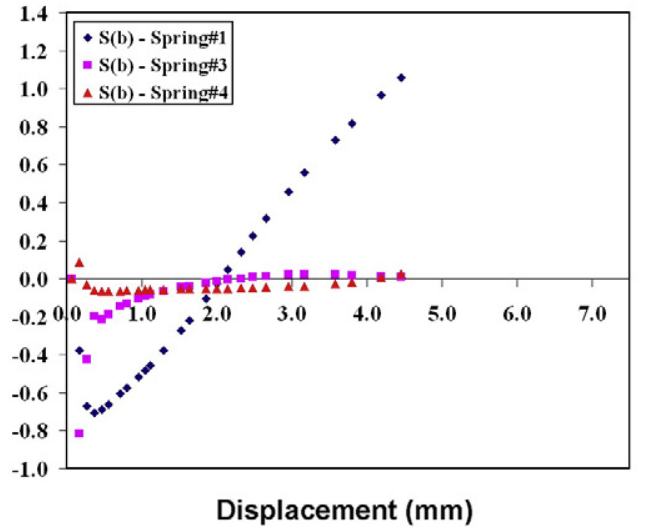

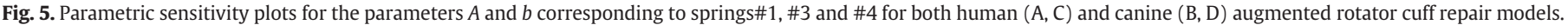
Both models appear to be most sensitive to perturbations in parameters $A$ and $b$ for spring\#1.

spring components in both human and canine rotator cuff repairs were most reliably modeled using the single-phase non-linear equation. The need for a biphasic equation to model the load-displacement behavior of the suture interface components may be due to the combined mechanisms of stretch of the tendon and slip of the suture from the tendon that occur in these components. The fact that spring\#4 of the human model was not best modeled by the biphasic non-linear equation as other suture interfaces may be due to the difference in the architecture of the human infraspinatus tendon compared to that of the canine infraspinatus tendon. Unlike the organized nature of collagen fibers of the canine infraspinatus tendon, the collagen fibers of the human infraspinatus tendon are more randomly organized, particularly in the region medial to the insertion site (Clark and Harryman, 1992; Dejardin et al., 1999). This random organization of the collagen fibers in the human tendon may minimize suture slip and thus explain why a single-phase non-linear equation was better for fitting the medial suture-tendon interface (spring\#4) of the human model.

Our study is not without limitations. First, compared to the clinical scenario, the experimental repairs used to develop our models were greatly simplified and idealized. An isolated tendon was released and repaired acutely, with only one type of surgical technique and one type of scaffold, and tested under only one loading condition. The model results are therefore dependent on the particular experimental conditions tested. While the scaffold and the surgical technique are representative of typical scaffolds and techniques commonly used for the repair of human rotator cuff tears, one must remain cautious in making a direct translation of these model predictions to human rotator cuff tendon repairs which are inherently multidimensional and structurally variable. Second, since the models are represented by non-linear-springs, there is dependence (cross-correlation) among the parameters for each component of the model. However, since the parameters for each spring were obtained from independent experiments, it is safe to assume that there is no dependence among parameters representing different springs. Therefore, assessing the relative contribution of each spring to the construct's performance by way of the sensitivity analysis is appropriate and any cross-correlation within the parameters of a given spring will not change these conclusions. Thirdly, we modeled mechanical testing performed under in vitro conditions, which may not reflect the biomechanics of in vivo repairs that are subjected to biological processes. Fourth, the model parameters for springs\#1 and 2 were obtained from failure testing of specimens that were first subjected to a cyclic loading protocol. Hence, the models cannot be used to predict the biomechanics of repairs on the first initiation of load following surgical repair. Finally, the analytical formulation used to predict the mechanical performance of the repairs is only valid up to and including the point of maximum (yield) load. Hence, the model cannot be used to predict failure loads of the repair.

In summary, we have developed and validated simple spring-based non-linear models for predicting the trends associated with scaffold augmentation of rotator cuff repairs. The ability of our models to predict the biomechanics of non-augmented and augmented rotator cuff repairs from both human and canine strengthens the interpretation, application and relevance of our observations. For the simplified repairs modeled herein, the total load was distributed $\sim 70-80 \%$ to the tendon repair component (i.e., tendon plus its suture attachment to bone) and $\sim 20-30 \%$ to the augmentation component (i.e., the scaffold plus its attachments to tendon and bone). This finding suggests that this particular scaffold, together with its attachments components, is less stiff than the tendon and its repair and highlights the applicability 
of the model to predict the degree of load sharing provided by scaffolds commonly used to augment rotator cuff repairs. The model results and sensitivity analysis suggest that although the scaffold contributes to the overall mechanical properties of the repair construct, the greatest improvements in the force carrying capacity of a tendon repair may be achieved by improving the properties of the bone-suture-tendon interface. In the future, we will use this model to conduct a parametric simulation study with the aim to predict the manner in which changes to the individual components of the repair, representing different surgical techniques and scaffold devices, may influence the biomechanics of the repair construct. The model provides, for the first time, a conceptual framework in which surgeons using scaffolds for augmenting tendon repair can understand their utility and apply them in a manner that maximizes their performance.

\section{Acknowledgments}

Financial support was provided by Cleveland State University (Molecular Medicine Fellowship for A. Aurora). Synthasome, Inc. (San Diego, CA, USA) donated the X-Repair devices used for obtaining experimental data for augmented, full-width rotator cuff repairs in canine cadavers.

\section{References}

Accousti, K.J., Flatow, E.L., 2007. Technical pearls on how to maximize healing of the rotator cuff. Instr. Course Lect. 56, 3-12.

Aurora, A., McCarron, J., Iannotti, J.P., Derwin, K., 2007. Commercially available extracellular matrix materials for rotator cuff repairs: state of the art and future trends. J. Shoulder Elbow Surg. 16, S171-S178.

Barber, F.A., Herbert, M.A., Boothby, M.H., 2008. Ultimate tensile failure loads of a human dermal allograft rotator cuff augmentation. Arthroscopy 24, 20-24.

Bartolozzi, A., Andreychik, D., Ahmad, S., 1994. Determinants of outcome in the treatment of rotator cuff disease. Clin. Orthop. Rel. Res. 308, 90-97.

Bishop, J., Klepps, S., Lo, I.K., Bird, J., Gladstone, J.N., Flatow, E.L., 2006. Cuff integrity after arthroscopic versus open rotator cuff repair: a prospective study. J. Shoulder Elbow Surg. 15, 290-299.

Boileau, P., Brassart, N., Watkinson, D.J., Carles, M., Hatzidakis, A.M., Krishnan, S.G., 2005. Arthroscopic repair of full-thickness tears of the supraspinatus: does the tendon really heal? J. Bone Joint Surg. Am. 87, 1229-1240.

Clark, J.M., Harryman, D.T., 1992. Tendons, ligaments, and capsule of the rotator cuff. Gross and microscopic anatomy. J. Bone Joint Surg. Am. 74, 713-725.

Cofield, R.H., Parvizi, J., Hoffmeyer, P.J., Lanzer, W.L., Ilstrup, D.M., Rowland, C.M., 2001. Surgical repair of chronic rotator cuff tears. A prospective long-term study. J. Bone Joint Surg. Am. 83, 71-77.

Coons, D.A., Alan, B.F., 2006. Tendon graft substitutes-rotator cuff patches. Sports. Med. Arthrosc. 14, 185-190.

Dejardin, L.M., Arnoczky, S.P., Clarke, R.B., 1999. Use of small intestinal submucosal implants for regeneration of large fascial defects: an experimental study in dogs. J. Biomed. Mater. Res. 46, 203-211.

Derwin, K.A., Codsi, M.J., Milks, R.A., Baker, A.R., McCarron, J.A., Iannotti, J.P., 2009. Rotator cuff repair augmentation in a canine model with use of a woven poly-L-lactide device. J. Bone Joint Surg. Am. 91, 1159-1171.
Derwin, K.A., Badylak, S.F., Steinmann, S.P., Iannotti, J.P., 2010. Extracellular matrix scaffold devices for rotator cuff repair. J. Shoulder Elbow Surg. 19, 467-476.

Funakoshi, T., Suenaga, N., Sano, H., Oizumi, N., Minami, A., 2008. In vitro and finite element analysis of a novel rotator cuff fixation technique. J. Shoulder Elbow Surg. 17, 986-992.

Galatz, L.M., Ball, C.M., Teefey, S.A., Middleton, W.D., Yamaguchi, K., 2004. The outcome and repair integrity of completely arthroscopically repaired large and massive rotator cuff tears. J. Bone Joint Surg. Am. 86, 219-224.

Gazielly, D.F., Gleyze, P., Montagnon, C., 1994. Functional and anatomical results after rotator cuff repair. Clin. Orthop. Rel. Res. 304, 43-53.

Gerber, C., Fuchs, B., Hodler, J., 2000. The results of repair of massive tears of the rotator cuff. J. Bone Joint Surg. Am. 82, 505-515.

Goutallier, D., Postel, J.M., Gleyze, P., Leguilloux, P., Van Driessche, S., 2003. Influence of cuff muscle fatty degeneration on anatomic and functional outcomes after simple suture of full-thickness tears. J. Shoulder Elbow Surg. 12, 550-554.

Hamada, K., Tomonaga, A., Gotoh, M., Yamakawa, H., Fukuda, H., 1997. Intrinsic healing capacity and tearing process of torn supraspinatus tendons: in situ hybridization study of alpha 1 (I) procollagen mRNA. J. Orthop. Res. 15, 24-32.

Harryman, D.T., Mack, L.A., Wang, K.Y., Jackins, S.E., Richardson, M.L., Matsen III, F.A 1991. Repairs of the rotator cuff. Correlation of functional results with integrity of the cuff. J. Bone Joint Surg. Am. 73, 982-989.

Iannotti, J.P., 1994. Full thickness rotator cuff tears: factors affecting surgical outcome. J. Am. Acad. Orthop. Surg. 2, 87-95.

McCarron, J.A., Milks, R.A., Iannotti, J.P., Derwin, K.A., 2010. Improved time-zero biomechanical properties using PLLA graft augmentation in a cadaveric rotator cuff repair model. Accepted for publication. J. Shoulder Elbow Surg.

Milgrom, C., Schaffler, M.B., Van Holsbeeck, 1995. Rotator-cuff changes in asymptomatic adults. The effect of age, hand dominance and gender. J. Bone Joint Surg. Br. 77 296-298.

Riley, G.P., Harrall, R.L., Constant, C.R., Chard, M.D., Cawston, T.E., Hazleman, B.L., 1994 Tendon degeneration and chronic shoulder pain: changes in the collagen composition of the human rotator cuff tendons in rotator cuff tendinitis. Ann. Rheum. Dis. 53, 359-366.

Romeo, A.A., Hang, D.W., Bach Jr., B.R., Shott, S., 1999. Repair of full thickness rotator cuff tears. Gender, age, and other factors affecting outcome. Clin. Orthop. Rel. Res. 367 243-255.

Sano, H., Wakabayashi, I., Itoi, E., 2006. Stress distribution in the supraspinatus tendon with partial-thickness tears: an analysis using two-dimensional finite element model. J. Shoulder Elbow Surg. 15, 100-105.

Sano, H., Yamashita, T., Wakabayashi, I., Itoi, E., 2007. Stress distribution in the supraspinatus tendon after tendon repair: suture anchors versus transosseous suture fixation. Am. J. Sports Med. 35, 542-546.

Seki, N., Itoi, E., Shibuya, Y., Wakabayashi, I., Sano, H., Sashi, R., et al., 2008. Mechanical environment of the supraspinatus tendon: three-dimensional finite element model analysis. J. Orthop. Sci. 13, 348-353.

Tempelhof, S., 1999. Age-related prevalance of rotator cuff tears in asymptomatic shoulders. J. Shoulder Elbow Surg. 8, 296-299.

Thomopoulos, S., Soslowsky, L.J., Flanagan, C.L., Tun, S., Keefer, C.C., Mastaw, J., et al., 2002. The effect of fibrin clot on healing rat supraspinatus tendon defects. J. Shoulder Elbow Surg. 11, 239-247.

Uhthoff, H.K., Trudel, G., Himori, K., 2003. Relevance of pathology and basic research to the surgeon treating rotator cuff disease. J. Orthop. Sci. 8, 449-456.

Vitale, M.A., Vitale, M.G., Zivin, J.G., Braman, J.P., Bigliani, L.U., Flatow, E.L., 2007. Rotator cuff repair: an analysis of utility scores and cost-effectiveness. J. Shoulder Elbow Surg. 16, 181-187.

Wakabayashi, I., Itoi, E., Sano, H., Shibuya, Y., Sashi, R., Mingawa, H., et al., 2002. Mechanical environment of the supraspinatus tendon: a two-dimensional finite element model analysis. J. Shoulder Elbow Surg. 12, 612-617.

Yamaguchi, K., 2001. Natural history of asymptomatic roator cuff tears: a longitudnal analysis of asymptomatic tears detected sonographically. J. Shoulder Elbow Surg. 10, 199-203. 